\title{
Effect of Mizagliflozin on Postprandial Plasma Glucose in Patients With Functional Constipation
}

TO THE EDITOR: We read the recent review by Fukui et $\mathrm{al}^{1}$ in this journal. In their paper, they referred that the bacterial product short chain fatty acid promotes glucose regulating hormones including glucagon-like peptide $1 .{ }^{1}$ We consider their review to be accurate and to provide further insight to the following phenomena.

Mizagliflozin, a novel sodium-glucose cotransporter 1 inhibitor, suppresses the absorption of glucose and water in the intestine. ${ }^{2,3}$ We reported that mizagliflozin showed favourable efficacy and safety for patients with functional constipation and the risk of hypoglycaemia would be low. ${ }^{4}$ However, the dynamic data of postprandial plasma glucose under the administration of mizagliflozin has not been shown yet.

A randomised open-label study which evaluated the effects of oral administration of mizagliflozin $5 \mathrm{mg}$ or $10 \mathrm{mg}$ once daily at 30 minutes after breakfast for 2 weeks on plasma glucose and spontaneous bowel movement in patients with functional constipation was conducted in Japan (ClinicalTrials.gov NCT02343978). Twenty five patients ( $5 \mathrm{mg}, \mathrm{n}=13$ and $10 \mathrm{mg}, \mathrm{n}=12$ ) were enrolled in the study. After 2-weeks, postprandial plasma glucose and insulin at 1 hour and 2 hours in $10 \mathrm{mg}$ of mizagliflozin and insulin at 2 hours in $5 \mathrm{mg}$ of mizagliflozin were significantly lower than those in the baseline (Table). Areas under the curves for plasma glucose and insulin were not changed by $5 \mathrm{mg}$ but significantly reduced by $10 \mathrm{mg}$ of mizagliflozin (glucose, $P=0.043$ and insulin, $P=0.007$, onesample $t$ test). No hypoglycemic symptoms were induced by mizagliflozin but one patient showed hypoglycemic value $(58 \mathrm{mg} / \mathrm{dL}$ ) of postprandial plasma glucose at 2 hours in $10 \mathrm{mg}$ of mizagliflozin. The mean number of spontaneous bowel movement per week significantly increased from 2.13 (SD, 0.45) at baseline to 7.99 (9.35) at week 2 in the $5 \mathrm{mg}$ group and from $1.76(0.62)$ to 6.81 (4.94) in the $10 \mathrm{mg}$ group.

These results suggest that mizagliflozin actually inhibits postprandial glucose absorption from the intestine and has insulinsparing effect. As Spiller ${ }^{5}$ previously commented, altered microbiota by the luminal glucose is one of factors affecting the effect of miza- gliflozin. Therefore, this study provides another evidence of the review by Fukui et al. ${ }^{1}$ This study also supports the concept of low risk of hypoglycemia by sodium-glucose cotransporter 1 inhibitors ${ }^{6}$, but low value of plasma glucose in rare cases should be recognized for further clinical studies.

Shin Fukudo ${ }^{1,2 *}$ and Kohei Kaku ${ }^{3}$ ${ }^{\prime}$ Department of Behavioral Medicine, Tohoku University Graduate School of Medicine, Sendai, Japan; ${ }^{2}$ Department of Psychosomatic Medicine, Tohoku University Hospital, Sendai, Japan; and ${ }^{3}$ Department of Internal Medicine, Kawasaki Medical School, Kurashiki, Japan

1. Fukui H, Xu X, Miwa H. Role of gut microbiota-gut hormone axis in the pathophysiology of functional gastrointestinal disorders. J Neurogastroenterol Motil 2018;24:367-386.

2. Ohno H, Kojima Y, Harada H, Abe Y, Endo T, Kobayashi M. Absorption, disposition, metabolism and excretion of $\left[{ }^{14} \mathrm{C}\right]$ mizagliflozin, a novel selective SGLT1 inhibitor, in rats. Xenobiotica 2019;49:463-473.

3. Inoue T, Takemura M, Fushimi N, et al. Mizagliflozin, a novel selective SGLT1 inhibitor, exhibits potential in the amelioration of chronic constipation. Eur J Pharmacol 2017;806:25-31.

4. Fukudo S, Endo Y, Hongo M, et al. Safety and efficacy of the sodiumglucose cotransporter 1 inhibitor mizagliflozin for functional constipation: a randomised, placebo-controlled, double-blind phase 2 trial. Lancet Gastroenterol Hepatol 2018;3:603-613.

5. Spiller R. Inhibiting glucose absorption to treat constipation. Lancet Gastroenterol Hepatol 2018;3:588-589.

6. Danne T, Biester T, Kordonouri O. Combined SGLT1 and SGLT2 inhibitors and their role in diabetes care. Diabetes Technol Ther 2018;20(suppl 2):S269-S277.

Financial support: This study was funded by Kissei Pharmaceutical Co, Ltd (Grant No. KWA1205).

Conflicts of interest: Shin Fukudo reports grants and personal fees from Kissei Pharmaceutical, during the conduct of the study; personal fees from Dainippon Sumitomo Pharma, grants and personal fees from Abott Japan, personal fees from Scampo Pharma, grants from Ono Pharmaceutical, grants and personal fees from Astellas Pharmaceutical, personal fees from Sanwa Chemical Co. Ltd, personal fees from Zeria, personal fees from Glaxo-Smith-Kline, personal fees 
Table. Changes in Plasma Glucose and Insulin Levels in the Meal Tolerance Test in the $5 \mathrm{mg}$ and $10 \mathrm{mg}$ Group

\begin{tabular}{|c|c|c|c|c|c|c|c|c|c|c|c|c|}
\hline Group & Weeks & Item & Times (hr) & $\mathrm{n}$ & Mean & $\mathrm{SD}$ & Min & Q1 & Median & Q3 & Max & $\begin{array}{c}P \text {-value } \\
\text { (week } 0 \text { vs } 2 \text { ) }\end{array}$ \\
\hline \multicolumn{13}{|l|}{ Plasma glucose $(\mathrm{mg} / \mathrm{dL})$} \\
\hline \multirow[t]{8}{*}{ Mizagliflozin $5 \mathrm{mg}$} & 0 & FPG & 0 & 13 & 94.2 & 11.1 & 79 & 87 & 92 & 100 & 114 & - \\
\hline & & PPG & 0.5 & 13 & 141.8 & 18.7 & 111 & 133 & 141 & 151 & 175 & - \\
\hline & & & 1 & 13 & 139.2 & 41.9 & 88 & 104 & 131 & 165 & 219 & - \\
\hline & & & 2 & 13 & 105.0 & 36.4 & 72 & 86 & 90 & 108 & 208 & - \\
\hline & 2 & FPG & 0 & 13 & 95.1 & 13.0 & 77 & 87 & 91 & 101 & 124 & 0.665 \\
\hline & & PPG & 0.5 & 13 & 140.3 & 32.0 & 109 & 116 & 135 & 156 & 210 & 0.797 \\
\hline & & & 1 & 13 & 125.8 & 48.2 & 81 & 98 & 110 & 129 & 231 & 0.216 \\
\hline & & & 2 & 13 & 91.0 & 20.3 & 71 & 78 & 87 & 89 & 134 & 0.064 \\
\hline \multirow[t]{8}{*}{ Mizagliflozin $10 \mathrm{mg}$} & 0 & FPG & 0 & 12 & 92.6 & 10.0 & 79 & 88 & 92 & 96 & 118 & - \\
\hline & & PPG & 0.5 & 12 & 143.4 & 31.0 & 107 & 121 & 141 & 150 & 206 & - \\
\hline & & & 1 & 12 & 129.0 & 45.0 & 81 & 100 & 109 & 154 & 243 & - \\
\hline & & & 2 & 12 & 105.8 & 28.5 & 76 & 84 & 100 & 124 & 163 & - \\
\hline & 2 & FPG & 0 & 12 & 93.4 & 7.2 & 83 & 89 & 93 & 97 & 111 & 0.659 \\
\hline & & PPG & 0.5 & 12 & 144.6 & 20.4 & 105 & 127 & 147 & 159 & 177 & 0.839 \\
\hline & & & 1 & 12 & 110.0 & 26.8 & 72 & 92 & 98 & 132 & 156 & 0.044 \\
\hline & & & 2 & 12 & 82.6 & 14.6 & 58 & 76 & 83 & 91 & 105 & 0.009 \\
\hline \multicolumn{13}{|l|}{ Insulin $(\mu \mathrm{U} / \mathrm{mL})$} \\
\hline \multirow[t]{8}{*}{ Mizagliflozin $5 \mathrm{mg}$} & 0 & Fasting & 0 & 13 & 5.28 & 2.24 & 2.7 & 3.8 & 4.9 & 5.6 & 10.1 & - \\
\hline & & After meal & 0.5 & 13 & 65.41 & 32.58 & 28.4 & 40.2 & 54.8 & 96.2 & 119.5 & - \\
\hline & & & 1 & 13 & 58.07 & 25.60 & 18.8 & 40.8 & 49.5 & 70.9 & 105.8 & - \\
\hline & & & 2 & 13 & 41.22 & 23.28 & 15.1 & 20.8 & 36.6 & 56.8 & 87.9 & - \\
\hline & 2 & Fasting & 0 & 13 & 5.75 & 3.09 & 1.8 & 3.7 & 5.5 & 6.6 & 11.1 & $0 \cdot 519$ \\
\hline & & After meal & 0.5 & 13 & 68.16 & 31.28 & 22.7 & 46.6 & 63.9 & 89.0 & 121.3 & $0 \cdot 504$ \\
\hline & & & 1 & 13 & 53.07 & 27.98 & 24.7 & 28.1 & 37.2 & 72.5 & 111.5 & 0.577 \\
\hline & & & 2 & 13 & 13.62 & 8.16 & 4.5 & 7.0 & 10.6 & 19.6 & 32.6 & $<0.001$ \\
\hline \multirow[t]{8}{*}{ Mizagliflozin $10 \mathrm{mg}$} & 0 & Fasting & 0 & 12 & 5.23 & 3.03 & 1.2 & 3.4 & 4.2 & 7.1 & 11.2 & - \\
\hline & & After meal & 0.5 & 12 & 79.03 & 74.08 & 21.8 & 37.5 & 58.1 & 93.6 & 296.0 & - \\
\hline & & & 1 & 12 & 56.49 & 28.44 & 31.5 & 39.3 & 44.4 & 68.4 & 130.3 & - \\
\hline & & & 2 & 12 & 35.34 & 12.58 & 18.1 & 23.3 & 36.5 & 45.5 & 57.7 & - \\
\hline & 2 & Fasting & 0 & 12 & 4.89 & 1.77 & 3.2 & 3.9 & 4.5 & 5.2 & 9.9 & 0.634 \\
\hline & & After meal & 0.5 & 12 & 72.98 & 50.39 & 32.2 & 39.2 & 55.4 & 77.7 & 190.7 & 0.582 \\
\hline & & & 1 & 12 & 39.88 & 17.11 & 18.8 & 21.6 & 39.9 & 55.1 & 64.9 & 0.021 \\
\hline & & & 2 & 12 & 8.92 & 2.85 & 5.0 & 6.7 & 8.0 & 12.0 & 13.5 & $<0.001$ \\
\hline
\end{tabular}

Min, minimum; Q, quartile; Max, maximum; FPG, fasting plasma glucose; PPG, postprandial plasma glucose.

At week 0 (without mizagliflozin) and 2 (with mizagliflozin), blood glucose and insulin levels were measured at 0, 30, 60, and 120 minutes after the patients ingested $431 \mathrm{kcal}$ standard breakfast of $67.2 \mathrm{~g}$ to $68.5 \mathrm{~g}$ of carbohydrates, $5.4 \mathrm{~g}$ to $5.6 \mathrm{~g}$ of fat, and $19.7 \mathrm{~g}$ to $21.1 \mathrm{~g}$ of protein.

$P$-values were calculated using the one-sample $t$ test.

from Mochida Pharmaceutical, personal fees from Shionogi Pharmaceutical, grants and personal fees from AstraZeneca, grants from Smoking Research Foundation, grants and personal fees from Tsumuta Co. Ltd., personal fees and non-financial support from Miyarisan Pharmaceutical, grants from Kao Co. Ltd., and grants from Zespri Co. Ltd, outside the submitted work. Kohei Kaku reports personal fees from Kissei Pharmaceutical, during the conduct of the study; grants and personal fees from Boehringer Ingelheim, grants from Daiichi Sankyo, grants and personal fees from Taisho Toyama Pharmaceutical, grants and personal fees from Mitsubishi Tanabe Pharma, grants and personal fees from Takeda Pharmaceutical, grants and personal fees from Astellas Pharma, personal fees from AstraZeneca, personal fees from Sumitomo Dainippon Pharma, personal fees from Kissei Pharmaceutical, personal fees from Kowa, personal fees from MSD, personal fees from Novartis Pharma, personal fees from Ono Pharmaceutical, and personal fees from Sanofi K.K, outside the submitted work.

Author contributions: Shin Fukudo and Kohei Kaku designed, performed, and analysed the study. All authors contributed to data interpretation, writing, and final approval of the manuscript. 